\title{
Médecine durable, facette d'une société durable
}

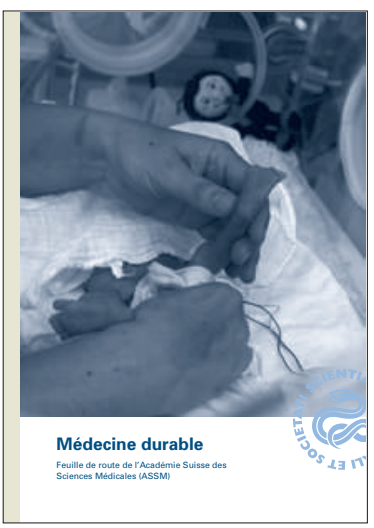

* A propos de santé publique: au moment où j'écris, peu après la réélection de Barack Obama, un leader républicain au Congrès annonce que son parti cesse sa guerre d'usure inepte contre la nouvelle loi de santé en disant: maintenant, «Obamacare is the law». Bonne nouvelle, s'agissant d'une loi qui diminue de 30 millions le nombre de personnes sans assurancemaladie.

1 ASSM. Feuille de route «Médecine durable». Bull Méd Suisses. 2012;93(45):1645-53.

2 Taverna E. De la valeur ajoutée de la «spiritualité». Bull Méd Suisses. 2012; 93(45):1678.

11 faut savoir gré à l'Académie suisse des sciences médicales d'avoir pris l'initiative d'un rapport sur la nécessité d'une médecine durable [1]. Rapport qui ne fera pas plaisir à tout le monde: quand on dit «le bénéfice des interventions médicales est souvent surestimé», «le système fixe des incitations fausses», «acquis corporatifs anachroniques», on sera critiqué pour auto-flagellation et défaitisme.

Un mérite de la feuille de route est sa perspective de santé publique, le fait de mettre notre métier, mélange d'artisanat, de science, d'art et de profit, selon le mot d'E. Taverna [2], carrément dans le contexte de la société*. Il est significatif qu'une instance estimée fasse un tel constat, jouant ainsi un rôle de donneur d'alerte (whistleblower).

Aussi compétents et perspicaces que soient les médecins, il est clair qu'ils ne sauraient à eux seuls effectuer les changements requis. Un grand mot dans les soins est aujourd'hui le partenariat, l'implication active du malade. Dans l'évolution souhaitée et avec toute une gamme de décideurs divers, il a des rôles à jouer: collaborer à l'optimisation du succès thérapeutique (pour diminuer les prestations inefficaces, ne pas les demander!), participer au débat politique pour un système sans incitations contreproductives et pour une planification sanitaire raisonnable. Cela veut dire éducation à la citoyenneté et au bon usage du système de santé, dès l'école. Cela veut dire aussi prise de conscience des enjeux à moyen-long terme, par exemple des effets indésirables ultérieurs de telle ou telle initiative attrayante dans l'immédiat.

Il conviendra que les politiciens soient jugés pour la qualité de leur action dans un sens prospectif, et leur vision, plutôt que démissionnés parce qu'ils annoncent ici et là des lendemains difficiles. Pour les élus, une telle attitude des citoyens permettrait de s'éloigner des promesses électorales surfaites; en général, on est loin du compte (mon quotidien titrait, à propos de la campagne électorale récente, «Une démocratie américaine minée par l'argent et le mensonge». Well...).

Il faut aussi prendre quelque recul, par exemple par rapport aux merveilles annoncées par la médecine améliorative. Il ne s'agit pas de rejeter les démarches de promotion d'un meilleur état de santé sous réserve de ce que derrière les discours est souvent présent, d'une manière ou de l'autre, le fantasme de l'immortalité (immortalité qui serait un cauchemar). Dans une optique de médecine durable, il faut prendre la mesure des risques de discrimination: ce seront les «best, brightest and richest» qui en bénéficieront surtout et il sera bien ardu de combler le fossé par rapport au patient lambda. De plus (horribile dictu), une médecine durable, «qui permette aussi aux générations futures de bénéficier de prestations satisfaisantes» (premier paragraphe du texte de l'ASSM) doit examiner d'un œil critique certaines perspectives liées au enhancement. Notamment parce que les générations futures (dont on rappelle qu'elles n'ont pas le droit de vote) ne seront pas bienvenues dans ce monde; en effet, elles accroîtront les pressions/empreintes sur le milieu de vie et leurs aînés, qui vivent si bien et si longtemps, n'apprécieront guère... Considérer avec l'Académie, «le caractère inabrogeable de la condition humaine comme ancrage de la vie entre la naissance et la mort». Soyons clairs: la durabilité voulue implique le remplacement et le renouveau - pas la fixation sur l'existant - y compris pour l'espèce humaine! Je ne sais plus qui a dit «Notre mortalité est la condition de notre liberté».

Durabilité a une dimension de raison, en tout cas de non-fuite précipitée en avant. La médecine durable ne peut être qu'une facette (importante) d'une société durable. Pour y arriver, simplement pas possible de s'en tenir encore et toujours à la mantra de la croissance. Un peu de retenue s.v.p. Ainsi, Barack Obama II fait espérer une contribution moins négligeable des Etats-Unisà la lutte contre le réchauffement climatique. Mais, même avec leur concours et celui espéré d'autres nations, freinera-t-on assez et assez vite?

La feuille de route liste de nombreuses mesures utiles. Beaux défis! Cela étant, une constante connue, au cabinet du médecin comme dans la vie de la res publica, c'est que les gens n'écoutent pas bien les nouvelles désagréables - et ce qu'écrit l'Académie sera ressenti désagréablement par ceux qui n'aiment pas l'idée de limites et de frugalité et ne veulent voir que la médecine triomphante qu'on imaginait au troisième quart du $\mathrm{XX}^{\mathrm{e}}$ siècle. Merci à celles et ceux qui en ont de mettre à disposition leurs baguettes magiques pour nous insuffler la volonté de devenir durables - et trouver les «solutions innovantes» que veut la feuille de route.

Jean Martin, membre de la Commission nationale d'éthique et de la Rédaction 